\title{
Acceptability of the Fitbit in behavioural activation therapy for depression: a qualitative study
}

Jenny Chum, ${ }^{1}$ Min Suk Kim, ${ }^{1}$ Laura Zielinski, ${ }^{2}$ Meha Bhatt, ${ }^{3}$ Douglas Chung, ${ }^{1}$ Sharon Yeung, ${ }^{1}$ Kathryn Litke, ${ }^{4}$ Kathleen McCabe, ${ }^{4,5}$ Jeff Whattam, ${ }^{4}$ Laura Garrick, ${ }^{4}$ Laura O'Neill, ${ }^{4,5}$ Stefanie Goyert, ${ }^{4}$ Colleen Merrifield, ${ }^{4}$ Yogita Patel, ${ }^{4}$ Zainab Samaan $3,4,5,6,7$

${ }^{1}$ Faculty of Health Sciences, McMaster University, Hamilton, Ontario, Canada; ${ }^{2}$ MiNDS Neuroscience Graduate Program, McMaster University, Hamilton, Ontario, Canada; ${ }^{3}$ Department of Health Research Methods, Evidence, and Impact, McMaster University, Hamilton, Ontario, Canada; ${ }^{4}$ Mood Disorders Research Unit, St. Joseph's Healthcare, Hamilton, Ontario, Canada; ${ }^{5}$ Department of Psychiatry and Behavioural Neurosciences, McMaster University, Hamilton, Ontario, Canada; ${ }^{6}$ Department of Psychiatry and Behavioral Neurosciences, Peter Boris Centre for Addictions Research, St. Joseph's Healthcare, Hamilton, Ontario, Canada; ${ }^{7}$ Department of Populations Genomics Program, Chanchlani Research Centre, McMaster University, Hamilton, Ontario, Canada

Correspondence to Dr Zainab Samaan, Department of Psychiatry and Behavioural Neurosciences, McMaster University, Hamilton, ON L8S 4K1, Canada; samaanz@mcmaster.ca

\section{ABSTRACT}

Introduction Major depressive disorder is characterised by low mood and poor motivation. Literature suggests that increased physical activity has positive effects on alleviating depression. Fitness-tracking devices may complement behavioural activation (BA) therapy to improve physical activity and mental health in patients with depression.

Objectives To understand patients' perceived benefit from the Fitbit and explore themes associated with patient experiences. To compare perceived benefit, patient factors, Fitbit usage and Beck's Depression Inventory (BDI) scores.

Methods Semistructured interviews were conducted with patients $(n=36)$ who completed a 28-week BA group programme in a mood disorders outpatient clinic. All patients were asked to carry a Fitbit One device. We conducted thematic analyses on the interviews and exploratory quantitative analyses on patient characteristics, Fitbit usage, steps recorded, perceived benefit and BDI scores.

Findings Twenty-three patients found the Fitbit helpful for their physical activity. Themes of positive experiences included self-awareness, peer motivation and goal-setting opportunities. Negative themes included inconvenience, inaccuracies and disinterest. Age, baseline and change in BDI scores, prior physical activity goals and familiarity with technology were not associated with perceived benefit from the Fitbit or usage. Perceived benefit was significantly $(p<0.01)$ associated with usage.

Conclusions Overall, the Fitbit is an acceptable tool to complement BA therapy for patients with depression. Many positive themes were concordant with current literature; however, patients also reported negative aspects that may affect use.

Clinical implications Clinicians and researchers should consider both strengths and limitations of activity trackers when implementing them to motivate patients with depression.

Trial Registration Number NCT02045771; Pre-results.

\section{BACKGROUND}

Depression is one of the highest contributors to the global burden of disease, ranking second in terms of years lived with disability. ${ }^{1}$ Moreover, depression has high costs associated with morbidity and care, costing the Canadian economy an estimated \$C32.3 billion in gross domestic product per year. ${ }^{2}$ It is also the most important risk factor for lifetime suicide attempts. ${ }^{3}$ Finally, individuals with depression are at greater risk of comorbidities such as cardiovascular disease and obesity. ${ }^{4}$

Physical activity has many benefits, including reduction in cardiovascular risk, body weight, visceral adiposity and improved cardiorespiratory fitness. ${ }^{5-7}$ Current literature suggests that physical activity can be impactful in managing depression. A randomised controlled trial (RCT) including 35 inpatients with depression found that addition of adjuvant exercise treatment can be helpful in improving depressive symptoms. ${ }^{8}$ Furthermore, a recent meta-analysis found exercise to have a significant effect on depression. ${ }^{9}$ Thus, interventions to improve physical activity may prove to be a valuable treatment addition in depression.

As technology continues to improve and become more integrated in our lives, new mobile health innovations are arising, with some aimed at improving physical activity. In particular, wearable activity trackers were shown to be helpful in improving physical activity and weight loss in the general population. ${ }^{10-12} \mathrm{~A}$ growing body of evidence has shown that such devices may be useful in clinical settings, such as cancer care. ${ }^{13} 14$ Moreover, recent studies have shown that wearable devices such as the Fitbit can measure step count, stairs climbed and distance travelled with comparable accuracy to a well-validated pedometer and other consumer-level activity trackers. ${ }^{15}$ The Fitbit is also capable of connecting data to personal online accounts, making it possible to collect aggregate data from these accounts given permission. As such, these devices may be useful in informing clinicians to improve management as well as for research purposes.

A small number of studies explored the use of such devices in mental health. A pilot study demonstrated the benefit of pedometer support in improving weekly step counts and overall mood for mild-tomoderate depression. ${ }^{16}$ One study of 10 patients with serious mental illness demonstrated that patients were receptive and accepting of these devices. ${ }^{17}$ Another exploratory study showed that increased steps measured on Fitbit wearable devices were associated with improved fitness and weight loss. ${ }^{18}$ Such evidence suggests that wearable activity tracking devices may have a place in mental health treatment; however, more evidence is required to understand their value, effectiveness and for whom it might be most beneficial.

\section{OBJECTIVES}

We examined (1) the proportion of participants who perceived benefit from the Fitbit and (2) themes that led to positive and negative experiences with Fitbits.

Secondary to this qualitative analysis, we aimed to perform exploratory quantitative analysis to examine (1) how participants' baseline characteristics related to perceived Fitbit benefit and use and (2) relationships between participants' perceived benefit and (a) Fitbit use and (b) depression severity. 


\begin{tabular}{|c|c|}
\hline Characteristic $(n=36)$ & \\
\hline Mean age (years) & $53.11^{*}$ (SD 12.35) \\
\hline Sex (\% female) & $18(48.7 \%)$ \\
\hline Mean baseline Beck's Depression Inventory score $\dagger$ & 36.27 (SD 11.43) \\
\hline Mean baseline body mass index (BMI) $\ddagger$ & 30.16 (SD 6.04) \\
\hline Prior smartphone use (\% using) & $23(63.9 \%)$ \\
\hline \multicolumn{2}{|l|}{ Education level } \\
\hline University & $13(36 \%)$ \\
\hline College/higher education & $13(36 \%)$ \\
\hline Secondary & $10(28 \%)$ \\
\hline
\end{tabular}

‡BMI scores are positively correlated with measures of body fat, where a BMI of 30 or higher indicates obesity.

\section{METHODS \\ Participants}

The Behavioural Activation Group Program in Patients with Depression (BRAVE) study, conducted at a mood disorders outpatient clinic in Hamilton, Ontario, Canada, is a pragmatic RCT to test the effectiveness of behavioural activation (BA) in depression. The study compares a BA intervention group to a waitlist control group receiving treatment as usual. Trained clinicians delivered BA to participants randomised to receive the intervention up to twice per week over 18 weeks, for a total of 28 sessions. Each session covered different topics of BA, such as value assessment, goal setting and leisure education. ${ }^{19} 20$

Patients (18 years or older) with major depressive disorder were approached to participate in the BRAVE programme. This included patients who were attending or referred for an assessment at the outpatient clinic, as well as patients who were referred by community family practices. All patients with depressive disorder receiving treatment for depression, including pharmacotherapy and psychotherapy, were eligible to participate. Exclusion criteria included inability to provide written informed consent, inability to understand written and spoken English and having a primary diagnosis other than depression.

Among the 87 participants who completed the BRAVE study, 36 participants completed a face-to-face interview with a research assistant. Baseline characteristics for the participants are shown in table 1.

\section{Procedure}

Fitbit One activity trackers were provided to all participants allocated to the BA group at the beginning of the BRAVE study and email accounts were created for each participant. The patients were encouraged to carry the Fitbit, embedded in a rubber clip, at all times to track their physical activity. At the beginning of each BA session, Fitbits were collected, recharged and synced to the online accounts of their respective holders by a research assistant. The participants' weekly activity data were then exported from the Fitbit website, and a personalised printout was generated including summaries of step count, stairs climbed and distance travelled. The printouts were shared with participants as they did not have access to the online accounts to minimise data manipulation. However, the Fitbit itself can show the number of steps taken daily, which can be seen by the patient.

\section{Data collection}

Qualitative data were collected through individual semistructured interviews with participants within 2 months of their completion of the BA

\section{Box Qualitative interview questions}

\author{
Main question \\ - How did you find the Fitbit? \\ - Was it helpful? \\ - What did you like about the Fitbit? \\ - What did you find challenging? \\ Other interview questions potentially related to Fitbit \\ - Do you have any other suggestions of changes that might be \\ made to improve the programme? \\ - What shouldn't they change? \\ - What was the most helpful part of the programme? \\ - Was there anything that you learnt or any activities you did in \\ the programme that you plan to continue on your own?
}

programme conducted by research assistants. The interviews lasted 30-60 min and were conducted either in person at the outpatient clinic or by telephone. All interviews were anonymised, voice recorded and transcribed verbatim. The interviews aimed to explore patients' experiences with their BA treatment and included a portion focused on their Fitbit use. Interviewers started with an open-ended question of 'how did you find the Fitbit?' and followed up further with probing questions when appropriate (box).

Additional data such as baseline Beck's Depression Inventory (BDI) scores, pretherapy goals, familiarity with technology and other patient characteristics were collected through questionnaires facilitated by a clinician during the study intake. Data pertaining to duration of Fitbit use were exported directly from participants' online accounts from the Fitbit website. BDI scores were collected weekly throughout the study including at completion.

\section{Qualitative analysis}

Two investigators (JC and MSK) independently analysed the transcriptions on Microsoft Excel to improve the breadth of perspectives. The investigators were educated in health sciences with prior clinical and research experiences. JC and MSK only interacted with patients during interviews. We based our approach on grounded theory due to its ability to systematically find in-depth themes.

After an initial round of thematic analysis, the investigators met to discuss and consolidate themes. This new list was used for a second round of independent coding. This was followed by a subsequent discussion between the investigators to reconcile conflicting findings. Sample saturation was discussed and established following the first round of analysis when no new themes emerged. The reporting of this study was in accordance with the Standards of Reporting Qualitative Research. ${ }^{21}$

\section{Exploratory quantitative analysis}

Exploratory analyses were conducted to examine how perceived benefit related to participants' baseline characteristics, including age, BDI scores, pretherapy physical activity goals and smartphone use, as well as per cent change in $\mathrm{BDI}$ scores from baseline to completion and Fitbit usage. All analyses were performed using IBM SPSS Statistics V.25. Participants were first sorted into one of two groups: those who perceived benefit and those who did not. Perceived benefit was defined as having found the Fitbit helpful as reported in the interviews. Those who were ambivalent were considered as not having found the Fitbit helpful and thus sorted into the latter group along with those who found the device unhelpful. Between group differences were calculated through independent t-tests for age, baseline BDI scores, per cent change in BDI scores from baseline to completion and Fitbit usage. Fitbit usage was determined by the period during which the participant used 
Table 2 Patient's perspectives and quotes

\begin{tabular}{|c|c|}
\hline \multicolumn{2}{|l|}{ Perceived helpfulness of the Fitbit } \\
\hline Helpful with physical activity $(n=23)$ & $\begin{array}{l}\text { "To be honest, the Fitbit and the steps... I'd say, jeez, I did } 20000 \text { today, let's get } 25000 \text { and that's usually getting out walking } \\
\text { the dogs". }\end{array}$ \\
\hline Not helpful with physical activity $(\mathrm{n}=7)$ & $\begin{array}{l}\text { "It was interesting to see but I don't know that it helped me. It didn't help me plan for anything and it didn't help me do more than I } \\
\text { would have anyway, but I loved looking at it". }\end{array}$ \\
\hline Helpful to others but not self $(n=4)$ & "I know when I'm active and when I'm not. I know what triggers it. Was it helpful for other people? For sure". \\
\hline \multicolumn{2}{|l|}{ Positives of the Fitbit } \\
\hline Peer motivation $(n=3)$ & "I was trying to get to the top, trying to beat the guy at the top. That was the only reason I was doing it". \\
\hline Goal setting $(n=5)$ & $\begin{array}{l}\text { "Well it motivated to move just a little bit more. If I was really close to my goal, and it helped me see during the day if I needed to } \\
\text { walk more or move more steps to get to my goal. So it just motivated me". }\end{array}$ \\
\hline Enjoyment $(n=13)$ & $\begin{array}{l}\text { "I actually enjoyed looking at it, through the day, when I was at home I would be out walking my dog at night and I'd take it out } \\
\text { and take a peek". }\end{array}$ \\
\hline \multicolumn{2}{|l|}{ Negatives of the Fitbit } \\
\hline Discouraging $(\mathrm{n}=2)$ & "It wasn't a tool for me. It wasn't a motivator for me at all. It just reinforced how much I wasn't doing". \\
\hline Disinterest $(n=10)$ & $\begin{array}{l}\text { "Sometimes I say, I knew I didn't' do it. So I got to do it. I tell myself, sometimes I say - Oh I don't need that thing to tell me what } \\
\text { to do". }\end{array}$ \\
\hline \multicolumn{2}{|l|}{ Plans for continued use } \\
\hline Plans to continue $(n=14)$ & "I plan to get a Fitbit or some similar activity monitor". \\
\hline No plans to continue $(n=3)$ & "I probably would have bought it but I didn't have the money at that time". \\
\hline Undecided ( $n=2)$ & "Possibly". \\
\hline
\end{tabular}

the Fitbit. The first week in which four or more days showed use, as reflected by the number of steps recorded, was marked as the start of the usage period. The last week in which four or more days showed use was recorded as the end of the usage period.

The two groups were examined through $X^{2}$ tests to determine if (a) self-reported pretherapy goals pertaining to physical activity and (b) familiarity with technology, as measured by previous smartphone use, were associated with perceived benefit.

Furthermore, to examine the relationships between baseline characteristics and Fitbit use, independent t-tests were used to compare physical activity goals and familiarity with technology with Fitbit usage as defined above. Pearson's correlation was employed to compare Fitbit usage with age and baseline BDI scores. Finally, Pearson's correlation was performed between average number of steps recorded on the Fitbit at week 3 of the study (when the majority of participants started using Fitbit) and baseline BDI scores.

\section{RESULTS}

\section{Perceived helpfulness of the Fitbit}

Patient responses were first categorised based on the helpfulness of the Fitbit as shown in table 2. Twenty-three patients indicated that the Fitbit was helpful, many of which indicating that it motivated them to be more active. Seven participants did not perceive the Fitbit to have influenced their physical activity. One participant indicated that even though the Fitbit did not improve his activity levels, he still enjoyed having it. Additionally, four participants reported that the Fitbit was not beneficial for themselves, but they acknowledged how it might be helpful to others.

Two participants did not provide a direct response in terms of perceived benefit from the Fitbit.

\section{Positive aspects of the Fitbit \\ Self-awareness}

The most significant theme that emerged was that the Fitbit provided participants with greater self-awareness of their physical activity. Twenty-seven participants enjoyed the step tracking and the immediate feedback about their physical activity. Of the 27 responses, 16 participants directly attributed this aspect to changes in their behaviour. In particular, the awareness of their lack of physical activity motivated them to increase their activity levels. Other participants discovered that they were more active than they believed, which encouraged them.

\section{Peer motivation}

Three of the participants identified peer motivation as factors contributing to their increased physical activity. Some participants found that comparing results with others inspired them to improve, while one participant was greatly motivated by the desire to be the best.

\section{Goal setting}

Five participants incorporated the immediate feedback aspect of the Fitbit into their goals - that is, they made daily or weekly step goals and used Fitbits to track their progress.

\section{Enjoyment}

Thirteen participants indicated that they enjoyed using the Fitbit, mostly attributing their enjoyment of the Fitbit to the aforementioned themes. One participant indicated that while the Fitbit did not increase his activity levels, he still loved to check it for his own information. Several 
participants also specifically enjoyed watching the growth of the 'flower' on the Fitbit display, which grew based on increases in physical activity.

\section{Negatives of Fitbit \\ Inconvenient}

Twelve participants found the Fitbit to be inconvenient. Many complained that the Fitbit was difficult to insert into its rubber clip, or that its small size caused it to be easily misplaced. Additionally, participants complained that the rubber of the Fitbit led to skin irritation, requiring alternative carrying methods. Moreover, as participants were not provided with their own Fitbit chargers, some complained that the battery would drain completely before their next BA session.

\section{Inaccurate}

Six participants felt that their Fitbits failed to accurately track progress during certain activities such as stair climbing or treadmill running.

\section{Discouraging}

Two participants found the Fitbit to be discouraging. One participant elaborated that the Fitbit highlighted her lack of activity, which only discouraged her further. In the other case, however, the participant felt this discouragement only initially and was eventually motivated by the Fitbit.

\section{Disinterest}

Ten participants reported having little interest in using the Fitbit. Some of these participants felt the Fitbit was unnecessary as they were already aware of their physical activity levels. Other participants reported that they were frustrated at the aforementioned negative elements.

\section{Plans for continued use}

Fourteen participants reported that they either already had or were seriously considering purchasing their own Fitbit or similar device. Two participants were unsure and three participants answered that they would not continue use-all indicating that price was the main deterrent.

\section{Exploratory quantitative analysis}

The results of the exploratory analysis are summarised in table 3.

\begin{tabular}{|c|c|c|c|}
\hline & Helpful $(n=23)$ & Not helpful $(n=13)$ & p Value \\
\hline $\begin{array}{l}\text { Mean Beck's Depression } \\
\text { Inventory (BDI) score* }\end{array}$ & 35 (SD 12.81) & 38.15 (SD 9.09) & 0.44 \\
\hline $\begin{array}{l}\text { Mean per cent change in } \\
\text { BDI scoret }\end{array}$ & $-21.51 \%$ (SD 33.91) & $-34.75 \%$ (SD 28.79) & 0.283 \\
\hline Mean age in years $\ddagger$ & 52.57 (SD 13.90) & 54.17 (SD 9.13) & 0.72 \\
\hline $\begin{array}{l}\text { Pretherapy physical activity } \\
\text { goals }\end{array}$ & 13 & 6 & 0.549 \\
\hline
\end{tabular}

\begin{tabular}{lllr} 
Prior smartphone use & 15 & 8 & 0.825 \\
Mean weeks used§ & 18.57 (SD 1.21) & 12.27 (SD 5.76) & $<0.001$ \\
\hline
\end{tabular}
${ }^{*} \mathrm{BDI}$ scores may range from 0 to 63 , where higher scores indicate higher severity
of depression.

†Five participants were excluded due to lack of data; three had found the Fitbit helpful.

$\ddagger$ One participant who found the Fitbit not helpful was excluded due to lack of age data.

§Four participants were excluded due to lack of data; two had found the Fitbit helpful.

\section{Perceived benefit}

No significant differences were found in age, baseline BDI scores, per cent change in $\mathrm{BDI}$ scores, pretherapy goals and smartphone use between those who did and did not find the Fitbit helpful.

Average age for those who found the Fitbit helpful was 52.57 (SD 13.90) years and 54.17 (SD 9.13) years for those who did not, $\mathrm{t}(33)=-0.359$, $p=0.72$. One participant was excluded from the analysis due to missing data.

Average baseline BDI score for those who found the Fitbit helpful was 35 (SD 12.81), while those who did not had a mean baseline BDI score of 38.15 (SD 9.09), $t(34)=-0.781, p=0.44$.

The mean per cent change in $\mathrm{BDI}$ score from baseline to completion of those who found the Fitbit helpful was $-21.51 \%$ (SD 33.91) compared with a change of $-34.75 \%$ (SD 28.79) for those who did not find it helpful, $t(29)=1.095, p=0.283$. Five participants were excluded due to missing data.

Of the 19 participants who reported physical activity-related goals during their baseline assessment, 16 found the Fitbit helpful. No relationship was found between having such goals and finding the Fitbit helpful, $X^{2}=0.358, p=0.549$.

Of the 23 participants who used smartphones, 15 found the Fitbit helpful. No relationship was found between smartphone use and finding the Fitbit helpful, $X^{2}=0.049, p=0.825$.

\section{Fitbit usage}

There was a significant relationship between total Fitbit usage and perceived benefit of Fitbit. The mean number of weeks of Fitbit use for those who found the Fitbit helpful was 18.57 (SD 1.21) and 12.27 (SD 5.76 ) weeks for those who did not, $t(30)=4.88, p<0.01$. On the other hand, there was no significant relationship between Fitbit use and physical activity goals, smartphone use or age.

The mean number of weeks of Fitbit use for those who had physical activity goals was 17.11 (SD 3.86) weeks, while those who did not have physical activity goals was 15.38 (SD 5.46), $t(30)=-1.047, p=0.303$.

The mean number of weeks of Fitbit use for those using smartphones was 16.95 (SD 4.03) weeks, while those who did not use smart phones was 15.62 (SD 5.33), $t(30)=-0.805, p=0.427$.

The correlation between age and Fitbit usage was not significant, $r(30)=0.194, p=0.287$. Likewise, the correlation between baseline BDI scores and Fitbit usage was also not significant, $r(30)=0.009, p=0.959$.

Four participants were excluded from the above Fitbit usage analysis due to missing data, two from the helpful and two from the not helpful group.

\section{Steps recorded}

There was no correlation between baseline BDI scores and average steps recorded on the Fitbit at week $3, r(34)=-0.009, p=0.960$. Two participants were excluded from the analysis due to missing data.

\section{CONCLUSIONS}

Of the 36 patients undergoing the BRAVE trial who completed the interviews, we found that the majority (64\%) of participants found the Fitbit to be helpful, identifying their Fitbit use as a factor in improving their physical activity level. In further elucidating perceptions of the Fitbit, various positive and negative aspects of the device were identified.

There were several positive themes related to Fitbit use. The most commonly identified was improved self-awareness, with many reporting a direct connection between this improved awareness and increases in physical activity. This is in concordance with literature on the general population which highlights improved awareness as the functional basis 
for these types of fitness devices. ${ }^{22}$ Other positive themes were goal setting, peer motivation and enjoyability. These findings are consistent with previous studies on healthy populations ${ }^{23}$ and those with mental illness. $^{17}$

We were able to identify challenges faced by participants when using the Fitbit. The two most reported barriers were the inconvenience and inaccuracies in the tracking feature. Previous studies had also found these themes to be barriers in both general populations and mental health settings using similar devices. ${ }^{1724}$ However, in the current study, comments on such barriers seemed more common, with nearly half of the participants quoting one or both concerns. In addition, some individuals found the Fitbit to be unnecessary as they were already aware of their activity levels, while others lost interest due to frustration stemming from a combination of these negative themes. That being said, these barriers did not appear to prevent the majority of the participants from enjoying and deriving benefit from the use of the Fitbit; in fact, many voiced plans to continue its use after the BA sessions. Finally, a potential challenge with Fitbit use was that it may further discourage users with low activity. While two individuals felt discouraged, one participant overcame this feeling and ultimately had a positive experience with the Fitbit. Thus, discouragement is unlikely to present as a common problem.

In an attempt to characterise who might be the most accepting towards the Fitbit, we compared age, baseline BDI scores, pretherapy goals relating to physical activity and familiarity with technology between those who found the Fitbit helpful and those who did not. We also explored these variables in relation to Fitbit usage. No significant relationships were found between these baseline characteristics and perceived benefit or Fitbit usage. These findings suggest that the Fitbit may be useful for patients with varying characteristics.

Additionally, we demonstrated that perceiving the Fitbit to be helpful was significantly related to an increased period of usage. This highlights the importance of ensuring the use of activity tracking for adequate durations and keeping the positive perceptions and barriers in mind as this may have significant impacts on physical activity and weight management in patients with depression. ${ }^{18}$

Interestingly, there was no significant relationship between perceived benefit of the Fitbit and per cent change in BDI scores. This contradicts previous literature supporting Fitbit use in treating depression. ${ }^{16-18}$ However, it is difficult to interpret these results given that the study was underpowered to test effectiveness and there were several confounders such as combined BA intervention.

Overall, these findings are exploratory and serve as an initial investigation into potential avenues to optimise treatment methods to incorporate activity tracking devices and other technology devices as indicated in the management of depression. These findings should be further studied to determine the effectiveness of activity tracking in managing depression.

\section{Limitations and suggestions}

This study was conducted in the setting of an outpatient BA programme. It would be interesting to see if other settings not including a BA component would yield similar findings. As well, since the interviews were not exclusively focused on the Fitbit, opportunities for further in-depth discussions might have been missed. As such, this study may have benefited from more focused discussions regarding Fitbits.

Another potential limitation is that the study restricted activity tracker usage to the Fitbit only, preventing participants from choosing their device of preference. In addition, participants were placed in a controlled environment of Fitbit use; they were not provided access to online accounts or Fitbit chargers, thus limiting the potential of the device. Such restrictions may have created differences in participants' perspectives compared with more pragmatic studies. For instance, the social aspect of the Fitbit seemed less significant than in prior studies, perhaps because patients were discouraged from sharing results. ${ }^{23}$ Moreover, as lack of motivation is often reported in individuals with depression, there is a possibility that disinterest in the Fitbit may be attributed to the low motivation and not reasons related to the device. ${ }^{25}$

Furthermore, it is uncertain whether participants who found the Fitbit helpful had actually increased their number of steps at study completion. As well, testing of effectiveness of the Fitbit in improving depressive symptoms was limited by the lack of a control group, relatively small sample size and the presence of confounders. In the future, larger studies with appropriate control groups are needed in order to explore whether Fitbit use affects number of daily steps or depression severity and whether this change is sustainable.

Lastly, this study and prior literature have focused on patients' perspectives of the Fitbit and similar activity trackers; however, the body of knowledge regarding clinician perspectives is much smaller. Potential next steps may be to determine if clinicians find activity trackers helpful in depression management-for instance, using them as goal-measurement devices.

\section{CLINICAL IMPLICATIONS}

While literature supports BA and exercise as a treatment for depression, BA is less represented in evidence-based mental health than other interventions. The findings of this paper aim to inform clinicians and researchers on the implementation of activity trackers in BA therapy in order to promote exercise. ${ }^{892026}$ The current study demonstrates that many patients with depression perceive the Fitbit as being beneficial to their physical activity. Those who found it helpful believed it to motivate them towards engaging in physical activity. However, barriers to Fitbit use suggest that individualised consultation may be warranted. This is further supported by the lack of associations between various patient characteristics and their perceived benefit.

Acknowledgements The authors would like to thank the BRAVE team and Out of the Blues programme. We would also like to thank the participants for taking the time to be part of this study.

Contributors JC, MSK and ZS conceived and designed the experiments. JC, MSK, $L Z, M B, D C$ and $S Y$ interviewed the participants and collected data. KL, KM, JW, LG, LO, SG, CM and YP recruited the patients, conducted the baseline interviews, developed Fitbit use manual and instructed the patients on Fitbit use. JC and MSK analysed the data and wrote the first draft of the manuscript. JC, MSK and ZS jointly developed the structure and arguments of the paper. All authors reviewed and approved the final manuscript.

Funding ZS (PI) was supported by Canadian Institutes of Health Research (CIHR) (Randomised Controlled Trials: Mentoring, code number 201303MTP-303860-182743) to conduct the BRAVE study. CIHR mentorship award (2013-2015) is to support PI research time.

\section{Competing interests None declared.}

Ethics approval The study was approved by the Hamilton Integrated Research Ethics Board (HIREB).

Provenance and peer review Not commissioned; externally peer reviewed.

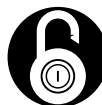

\section{OPEN ACCESS}

Open Access This is an Open Access article distributed in accordance with the Creative Commons Attribution Non Commercial (CC BY-NC 4.0) license, which permits others to distribute, remix, adapt, build upon this work non-commercially, and license their derivative works on different terms, provided the original work is properly cited and the use is non-commercial. See: http://creativecommons.org/licenses/ by-nc/4.0/

\section{doi:10.1136/eb-2017-102763}

Received 15 June 2017; Revised 15 September 2017; Accepted 18 September 2017 


\section{REFERENCES}

1. Murray CJ, Lopez AD. Measuring the global burden of disease. N Engl J Med 2013;369:448-57.

2. Southerland G, Stonebridge C. 2016. Healthy brains at work: estimating the impact of workplace mental health benefits and programs. Ottawa:The Conference Board of Canada. http://www.conferenceboard.ca/e-library/abstract.aspx?did=8242 laccessed 8 Jun 2017).

3. Miret M, Ayuso-Mateos JL, Sanchez-Moreno J, et al. Depressive disorders and suicide: epidemiology, risk factors, and burden. Neurosci Biobehav Rev 2013;37:2372-4.

4. Chapman DP, Perry GS, Strine TW. The vital link between chronic disease and depressive disorders. Prev Chronic Dis 2005;2:A14.

5. Bartels SJ, Pratt SI, Aschbrenner KA, et al. Clinically significant improved fitness and weight loss among overweight persons with serious mental illness. Psychiatr Serv 2013;64:729-36.

6. Kahl KG, Kerling A, Tegtbur $U$, et al. Effects of additional exercise training on epicardial, intra-abdominal and subcutaneous adipose tissue in major depressive disorder: a randomized pilot study. J Affect Disord 2016;192:91-7.

7. Stubbs B, Rosenbaum S, Vancampfort D, et al. Exercise improves cardiorespiratory fitness in people with depression: a meta-analysis of randomized control trials. J Affect Disord 2016;190:249-53.

8. Legrand FD, Neff EM. Efficacy of exercise as an adjunct treatment for clinically depressed inpatients during the initial stages of antidepressant pharmacotherapy: an open randomized controlled trial. J Affect Disord 2016;191:139-44.

9. Schuch FB, Vancampfort D, Richards J, et al. Exercise as a treatment for depression: a meta-analysis adjusting for publication bias. J Psychiatr Res 2016;77:42-51.

10. Wang JB, Cadmus-Bertram LA, Natarajan L, et al. Wearable sensor/device (Fitbit One) and sms text-messaging prompts to increase physical activity in overweight and obese adults: a randomized controlled trial. Telemed J E Health 2015;21:782-92.

11. Shuger SL, Barry VW, Sui $X$, et al. Electronic feedback in a diet- and physical activity-based lifestyle intervention for weight loss: a randomized controlled trial. Int $J$ Behav Nutr Phys Act 2011;8:41.

12. Cadmus-Bertram LA, Marcus BH, Patterson RE, et al. Randomized trial of a fitbit-based physical activity intervention for women. Am J Prev Med 2015;49:414-8

13. Gell NM, Grover KW, Humble M, et al. Efficacy, feasibility, and acceptability of a nove technology-based intervention to support physical activity in cancer survivors. Support Care Cancer 2017;25:1291-300.
14. Rosenberg D, Kadokura EA, Bouldin ED, et al. Acceptability of Fitbit for physical activity tracking within clinical care among men with prostate cancer. AMIA Annu Symp Proc 2016;2016:1050-9.

15. Case MA, Burwick HA, Volpp KG, et al. Accuracy of smartphone applications and wearable devices for tracking physical activity data. JAMA 2015;313:625-6.

16. Kerr J, Calfas KJ, Caparosa S, et al. A pilot study to assess the feasibility and acceptability of a community based physical activity intervention (involving internet, telephone, and pedometer support), integrated with medication and mood management for depressed patients. Ment Health Phys Act 2008;1:40-5.

17. Naslund JA, Aschbrenner KA, Barre LK, et al. Feasibility of popular m-health technologies for activity tracking among individuals with serious mental illness. Telemed J E Health 2015;21:213-6.

18. Naslund JA, Aschbrenner KA, Scherer EA, et al. Wearable devices and mobile technologies for supporting behavioral weight loss among people with serious mental illness. Psychiatry Res 2016;244:139-44.

19. Samaan Z, Dennis BB, Kalbfleisch L, et al. Behavioral activation group therapy for reducing depressive symptoms and improving quality of life: a feasibility study. Pilot Feasibility Stud 2016;2:e22.

20. Samaan Z, Litke K, McCabe K, et al. A pragmatic pilot randomized trial to investigate the effectiveness of behavioural activation group therapy in reducing depressive symptoms and improving quality of life in patients with depression: the BRAVE pilot trial protocol. Pilot Feasibility Stud 2015;1:39.

21. O'Brien BC, Harris IB, Beckman TJ, et al. Standards for reporting qualitative research. Academic Medicine 2014;89:1245-51.

22. Consolvo S, Klasnja P, McDonald D, et al. Flowers or a robot army? Encouraging awareness \& activity with personal, mobile displays. Proceedings of the 10th international conference on Ubiquitous computing-UbiComp '08 2008, 2008: 54-63

23. Fritz T, Huang E, Murphy G, et al. Persuasive technology in the real world: a study of long-term use of activity sensing devices for fitness. Proceedings of the 32nd annual ACM conference on Human factors in computing systems-CHI '14 2014, 2014: 487-96

24. Fausset CB, Mitzner TL, Price CE, et al. Older adults' use of and attitudes toward activity monitoring technologies. Proc Hum Factors Ergon Soc Annu Meet 2013;57:1683-7

25. Searle A, Calnan M, Lewis G, et al. Patients' views of physical activity as treatment for depression: a qualitative study. Br J Gen Pract 2011;61:149-56.

26. Ekers D, Webster L, Van Straten A, et al. Behavioural activation for depression; an update of meta-analysis of effectiveness and sub group analysis. PLoS One 2014;9:e100100 